\title{
¿Cómo influye la educación ambiental en la cultura?
}

\section{¿How does environmental education influence in the culture?}

\author{
Por: Gustavo Adolfo Bonilla Pérez ${ }^{1}$ y Bladimir Vera Marín²
}

Recibido: 22 -03- 2011

Aceptado: 07-07-2011

\section{Resumen}

La revisión de literatura muestra a los problemas ambientales como problemas sociales, en las cuales la educación debe de tomar papel fundamental relacionando las prácticas educativas con la realidad, igualmente, por las prácticas culturales las cuales parecen no partir de una actitud hacia el cuidado del entorno, en tanto se encuentran enmarcadas e influenciadas por un "conjunto de las creencias, actitudes, normas y valores que tiene como objeto de atención el medio ambiente en su conjunto o aspectos particulares del mismo, tales como la escasez de recursos naturales, la disminución de especies, la degradación de espacios naturales o la percepción e impacto de las actividades humanas" (Corraliza, 2001).

La propuesta de investigación surge de observar que la "educación ambiental" procedente de la escuela, de entornos no-formales e informales, no se vuelve acción; es decir, nuestro comportamiento no se hace parte de la cultura (González, 1999). Además, se plantea cómo analizar las relaciones que establecen entre: ambiente, cultura y educación.

Se puede concluir que las relaciones educación, cultura y ambiente, afecta inicialmente a la escuela, haciendo pensar, que las prácticas escolares tendrían que incluir propuestas didácticas dirigidas a configurar relaciones consistentes entre educación ambiental y comportamiento frente al medio.

Palabras Claves: Educación, cultura, ambiente, educación ambiental.

\section{Abstract:}

The literature review shows environmental problems as social ones, in which education must assume an important role relating educational practices with the

\footnotetext{
${ }^{1}$ Docente Instituto San Carlos de la Salle, (tavobon@yahoo.com). Licenciatura en Educación Básica Énfasis Ciencias Naturales y Educación Ambiental, Facultad de Educación, Universidad de Antioquia

2 Estudiante maestría en bosques y conservación ambiental, Universidad Nacional sede Medellín (bladoo385@hotmail.com). Licenciatura en Educación Básica Énfasis Ciencias Naturales y Educación Ambiental Facultad de Educación, Universidad de Antioquia
} 
reality as well as due to the cultural practices that appear not to start from a careful attitude toward the environment, because they are in line and influenced by a set of beliefs, attitudes, regulations and values which focus on the environment as a whole or its particular aspects such as the lack of natural resources, species decrease, natural spaces degradation or the perception oor impact of human activities ( Corraliza, 2011).

The research proposal arises from observing that the 'environmental education' that comes from the school, in informal and non-formal settings, doesn't become action; in other words, our behavior is not part of the culture (Gonzalez, 1999). Also it is set how to analyze the relationships that are established among environment, culture and education.

To conclude, the relationships among education, culture and environment, initially affects the school, suggesting that school practices should include educational proposals aimed at setting a consistent links between environmental education and behaviour towards the environment.

Keywords: Education, Culture, Environment, Environmental Education.

En la actualidad, la educación se ha alejado en gran proporción de su verdadero objetivo como ente formador de ciudadanos autónomos e íntegros, capaces de vivir en armonía con su entorno. Hoy en día, sólo se preocupa por "enseñar" conceptos y un sin número de teorías que ayudan muy poco a la toma de una conciencia y de una acción ambiental de equilibrio que beneficie en igual medida a ambas esferas: la social y la natural.

Por otro lado, la educación es la fuente de todo conocimiento y la responsable de la formación de una cultura porque tiene como propósito, formar un ser humano integral y culturalmente capaz de vivir en armonía con su medio social y principalmente con su medio natural.

Nuestro entorno, es de una singular riqueza en recursos naturales, aunque el hombre en su afán de someter a la madre tierra bajo su dominio se ha ensañado son su degradación constante y sin ningún tipo de reparo, conllevando a su destrucción continua, generando graves problemas ambientales e incluso sociales manifestados hoy día con cambios climáticos, desaparición de especies vegetales y animales. Además de lo anterior, esto no es más que el inicio de una cadena preocupante de acontecimientos naturales, sociales y culturales que ponen en riesgo la integridad de todo ser viviente en el futuro.

En palabras textuales del PRAE del Centro Educativo Minuto de Dios?

\footnotetext{
${ }^{3}$ Proyecto Ambiental Escolar -PRAE-. “Una estrategia pedagógico - ambiental para la convivencia ciudadana". Ciencias Naturales y Educación Ambiental. Centro Educativo Minuto de Dios. Montería. 2008
} 
"Ante esta situación ambiental en nuestro país, tanto a nivel rural como urbano, los maestros tenemos un gran reto pedagógico: el fomento y la creación de una cultura ambiental, mediante estrategia pedagógica en el proceso educativo y a partir del proyecto de vida de cada individuo y comunidad, de tal manera que se oriente y se armonice la relación de convivencia social... y con el entorno biofísico"

Siguiendo con lo anterior, se abre la necesidad de pensar la educación ambiental en términos de transformación cultural, por lo cual, se establece la siguiente pregunta ¿cómo influye la educación ambiental en la cultura?: teniendo como punto de apoyo, que la escuela en cuanto sistema social y democrático, debe educar para que los individuos y las colectividades comprendan la naturaleza compleja del ambiente, resultante de la interacción de sus aspectos biológicos, físicos, químicos, sociales, económicos y culturales; construyan valores y actitudes positivas para el mejoramiento de las interacciones hombre - sociedad - naturaleza, para un manejo adecuado de los recursos naturales y para que desarrollen las competencias básicas para resolver problemas ambientales (Lineamientos curriculares. Ministerio de Educación Nacional, 1998).

Por consiguiente, se debe de comenzar a comprender el sistema educativo que, hoy día es un sistema complejo, transversal, interdisciplinario e interdependiente, conformado por la familia, la sociedad y la cultura y, de acuerdo a lo anterior, se estaría hablando de educación formal, educación informal y educación no formal. Evidenciando una nueva concepción de educación, la cual deja de ser un fin de cuatro paredes -salón de clases- y trasciende sus horizontes hacia el exterior, donde sus influencias y certezas, se pueden ver en el mundo de la vida de cada estudiante, a través de su interacción con el contexto cultural y social, obteniendo así, un importante papel en la cotidianidad, la realidad y en las decisiones que tomen dentro de ellas. Es hora de pensar sobre la trascendencia del papel docente como eje integrador, orientador y dinamizador de los procesos de enseñanza y aprendizaje, en el contexto de cada estudiante, cuyo papel sea centrado en propuestas y acciones en pro del cuidado ambiental. Igualmente, sin separar la íntima relación entre la esfera social, ambiental, cultural, etc.

Para tal fin, se hace necesario conocer el mundo interno de cada persona; en cuanto a sus pensamientos, sus ideales, su conciencia y comportamiento ambiental; cualidades intrínsecas que al ser humano posibilitan potenciar un metaconocimiento, al reconocimiento de conductas adecuadas o inadecuadas dentro del entorno. Por tal motivo, se considera que los procesos de enseñanza y aprendizaje deben de interactuar con el mundo social y ambiental de los estudiantes, con el propósito de iniciar la construcción de objetivos en relación con la academia y la realidad ambiental actual, para propender de esta forma, por un conocimiento significativo.

Sin embargo, la educación junto a su proceso de formación, se encuentra sumergida dentro de un tiempo y un espacio determinado. Por lo tanto, la enseñanza en torno a la problemática ambiental, inicia su estructuración desde unas características específicas de acuerdo con las necesidades, conciencia y comportamiento ambiental de las personas como principales actores que influyen en la construcción o destrucción del mundo natural. A sí mismo, la educación se enmarca dentro de un sistema en el cual interactúa 
el ambiente, la cultura y la sociedad, dando cuenta de esta forma, de la existencia inevitable entre la esfera educativa y la realidad social y ambiental actual.

Siguiendo a González (1999) la cultura es la síntesis de procesos biológicos y conductuales y está representada por: tecnología, organización de los procesos de producción y reproducción, elementos cognoscitivos y simbólicos al igual que objetos materiales. La crisis ambiental está estrechamente relacionada con la conformación del mundo educativo y social, tratando que las actitudes que se aprenden en la escuela, sean practicadas en la sociedad, en este caso, un comportamiento en beneficio del ambiente, sin dejar de lado, que en muchos actos del ser humano, no conllevan a una actividad que ayude al ambiente a mantenerse en forma sustentable y quizás, se vuelvan costumbres convirtiéndose en estilos de vida en las personas. Por otra parte "Cultura es sobre todo, la formación integral de la personalidad, es todo lo que el hombre realiza en concreto para dominar la naturaleza, las iniciativas que adopta para modificar el medio ambiente natural, para transformar la sociedad en la satisfacción de necesidades individuales y colectivas, y a sus valores...cultura significa ante todo, desarrollo armónico y completo del hombre, buen gusto, sensibilidad y finura la entender y en el valora" (Andreu Y Hernández, 1999); en donde no solo se observa una visión externa del mundo y su relación entre las esferas sociales y simbólicos, sino en la interpretación del mundo que cada persona tiene en su interior, como se expresa y como puede modificar ese medio partiendo de sus concepciones, las cuales muchas son aprendidas en la escuela.

Partimos de que es el hombre el mayor agente transformador y modelador del mundo natural, por lo tanto, debemos analizar la influencia interactiva de las variables actitudinales y contextuales sobre la conducta ambiental y el sentimiento subjetivo de obligación moral frente al ambiente. Así entonces, asumimos que son problemas cuyas raíces se encuentran en la relación entre el ser humano y el mundo ambiental. No obstante, se encuentra supeditado a sus conocimientos -educación - a su ética, a su moral y a su comportamiento de ser supremo, dominador de todo cuanto existe en una cultura.

Visto así, compartimos con Mahecha (2003) que [...] se identifica a la educación ambiental como un problema, ya que encontrándose construida sobre la plataforma del deber ser, se observa y se entiende como una materia más con contenidos específicos al interior del currículo y no como un nuevo enfoque holístico dentro del quehacer educativo. Lo importante es que se siga formando en educación ambiental, como seres inmersos en una cultura, donde muestran esa formación con sus actitudes y estilos de vida propios.

La escuela tiene gran responsabilidad en la educación ambiental de las personas, pero al parecer los profesores la conciben de forma equivocada. No es sólo cuestión de PRAES ó PROCEDAS4, pues suelen generarse sentimientos momentáneos. Se plantea que la educación ambiental no se cierre a la construcción de propuestas sobre "reciclaje" ó "limpiemos la quebrada todos juntos". Consideramos la importancia de trascender, para que nuestros estudiantes cambien su forma de ver el medio, pensando en

\footnotetext{
${ }^{4}$ En nuestro contexto PRAE significa Proyecto Ambiental Escolar y PROCEDA hace referencia a Proyecto Ciudadano de Educación Ambiental.
} 
cambiar nuestra vida desde lo cotidiano, lo cual es más próximo a nuestro ambiente. La responsabilidad de los maestros está en toda la sociedad, para generar cambios en la existencia de todos, con una perspectiva de bienestar social.

La educación actual está pues descontextualizada de la realidad del entorno, porque sólo se encuentra direccionada hacia la adquisición de teorías, conocimientos y conceptos teóricos, olvidando la formación integral de los estudiantes cuyo resultado se evidencia en comportamientos que en nada favorecen al ambiente.

Todo lo anterior se vincula con un buen propósito plantado por Ruvalcaba (2002) Es conveniente que el alumno adquiera la capacidad de cuestionarse y de cuestionar su realidad, sólo de esta manera pensará, le surgirán dudas que lo lleven a plantear problemas de investigación y por ende a la búsqueda de soluciones a la problemática ecológica de su entorno. Es trascendental vincular el aprendizaje desde cualquier lugar, con la realidad del entorno, es decir, donde el proceso de aprendizaje tenga como pilares a la sociedad, a la cultura y el fomento en el sujeto en formación por el cuestionamiento de su mundo, de su realidad. Por lo tanto, se torna importante, la trascendentalidad de vincular el aprendizaje con la realidad, donde el estudiante busque una aplicación práctica en su entorno, de esta manera, algún día podremos encontrar soluciones a los grandes problemas ambientales. El reto está centrado pues, en transformar los salones de clases en aulas abiertas contextualizadoras de un saber específico en conocimientos significativos y de acción.

Entonces, ¿Qué puede estar pasando? Se hace alusión constantemente que...

..."una de las respuestas a la crisis ambiental ha sido la educación ambiental, ya que las ciencias de la educación, se ocupan del proceso formativo del hombre, del desarrollo del mismo, es decir, del cómo éste se prepara a lo largo de su vida para interactuar con el medio ambiente, esta educación debe promover la formación de una conciencia ambiental en los seres humanos que les permita convivir con el entorno, preservarlo, y transformarlo en función de sus necesidades, sin comprometer con ello la posibilidad de las generaciones futuras de satisfacer las suyas, de preservar y desarrollar la riqueza cultural de la humanidad, de producir bienes y riquezas materiales, incrementar el potencial productivo, asegurando oportunidades equitativas para todos, sin que ello implique poner en peligro nuestro ambiente, incluidos sus diferentes sistemas del mismo" (Alea, 2008.).

De igual forma y estando de acuerdo con Alea (2008):

"La educación ambiental debe entenderse como un proceso de aprendizaje que debe facilitar la comprensión de las realidades del medioambiente, del proceso socio histórico que ha conducido a su actual deterioro; que tiene como propósito que cada individuo posea una adecuada conciencia de dependencia y pertenencia con su entorno, que se sienta responsable de su uso y mantenimiento, y que sea capaz de tomar decisiones en este plano".

En otras palabras, socialmente la educación ambiental no está teniendo procesos correspondientes con la realidad actual, ni con las actitudes de cada persona formada en una cultura. Por consiguiente, el papel del educador no trasciende más allá de 
una capacitación o de una clase y es raro que llegue al ciudadano del común. ¿Qué está pasando? Se cree que los procesos de formación en educación ambiental no se están elaborando de manera que repercuta en la sociedad, causando problemas ambientales "el reconocimiento de la problemática ambiental y la reflexión sobre sus causas, se constituyen en un detonante que evidencia el conflicto naturaleza-cultura, que ha acompañado todo el desarrollo de la modernidad y la construcción de la sociedad industrial avanzada" (González, 1999).

"Desde el punto de vista de la información y el conocimiento, un aula es similar a un sistema cerrado; la información entra al entorno con el ingreso docente, y, como a sido documentado ampliamente, los conocimientos sirven únicamente para solucionar problemas escolares" (PRAE, Op. Cit. 2008). La escuela es el lugar donde se crea y transforma el conocimiento de cada individuo, por lo tanto, es conveniente que la vida, la naturaleza y el entorno biofísico entren en ella como pilares de estudio, reflexión e intervención; asimismo de lograr la construcción de un saber ambiental significativo.

Se sabe de antemano que el sistema educativo ha permanecido casi inmodificable durante mucho tiempo, sólo ha hecho pequeños ajustes con respecto al tiempo y al espacio en el cual se desarrolla, pero no ha contado con grandes y trascendentales cambios que garanticen la comprensión de los saberes allí presentados. Aún vemos que nos estamos quedando en aspectos netamente teóricos, los cuales no trascienden las fronteras del aula, no llegando de esta forma a transformar la vida social y cultural del ser humano.

La nueva educación debe formar ciudadanos críticos, reflexivos, activos y transformadores de su entorno, específicamente, de su entorno ambiental, que les permita actuar de la mejor manera en pro tanto de su bienestar, como del bienestar del ambiente natural. Somos el resultado de una interacción compleja entre sociedadcultura-naturaleza, en donde la educación no debe desconocerla, por lo cual debe actuar acorde a esta circunstancia, pues somos sólo un elemento de un gran tejido; actuamos para y a partir de una cultura que se encuentra determinada por la educación, la sociedad, la política y la economía. Sería un logro significativo posibilitar la integración de la academia con el estudiante y su entorno biofísico, con el fin de iniciar la reflexión, toma de conciencia y de acción en la solución de problemas ambientales, rompiendo los límites existentes, arraigados entre educación formal, no formal e informal.

"El problema que aún sigue abierto, consiste no tanto en definir y describir la preocupación ambiental, sino, sobre todo, de explicar los distintos niveles de conciencia ambiental en función de variables sociales, psicológicas y de visiones del mundo" (Corraliza, 2001). Esto sería un trabajo riguroso pero que se puede leer fácilmente en diferentes contextos sociales, función correspondiente a ramas como la psicología, la sociología y/o la antropología, sin descartar el campo educativo.

Pareciera que los problemas ambientales tuvieran su mejor (única) solución en la esfera de la técnica y de las Ciencias Naturales, mas no en las transformaciones éticas, sociales y en los cambios de comportamiento -cultura- de los individuos. Sabemos que por definición, los problemas ambientales exigen un tratamiento transversal e 
interdisciplinario. Los hechos responsables de una ética ambiental y social deben ser colectivos e interdisciplinares mediante la integración de las diversas áreas del saber conllevando a enfrentar la problemática ambiental, desde todas las perspectivas posibles abarcando lo social, político, económico, cultural y educativo. Pero toda la culpa no es de aquel que nos forma, es responsabilidad de aquellos que estamos allí sentados, con actitud pasiva sin capacidad de proponer y emprender cambios significativos en nuestro entorno; iniciando tal vez con obras pequeñas, pero que pueden desde luego convertirse en hechos reales.

La educación ambiental debe de formar para una mejor calidad de vida sin comprometer las generaciones futuras; diariamente nos enseñan este principio del desarrollo sostenible, pero los grandes empresarios y las grandes potencias no se están comprometiendo con el cuidado del ambiente. Sin embargo, es hora de repensar esto; es toda la sociedad la que tiene directo compromiso con la causa y la solución de los problemas ambientales, se debe de comenzar por casa, con estilos de vida que no comprometan el ambiente.

En cuanto a lo anterior, muchos estudios actuales en torno a la realidad holística del hombre plantean la necesidad de una integración mayor entre lo objetivo y lo subjetivo, entre lo comportamental y lo cognoscitivo, entre lo experimental y lo social; esto es, entre las disciplinas que apuntan a lo natural y a lo social.

El fin de integrar conceptos a nuestra realidad social es el reto de la moderna visión de la investigación para brindar una solución acorde con la problemática social y ambiental actual, entendiendo sin embargo, lo que aquí se pretende, es poder ligar al individuo y su comportamiento grupal y colectivo con su medio natural y social como un contexto de interacción que busca el equilibrio. De acuerdo a lo anterior como lo hace explícito Ramírez, en su libro Ética Ambiental (1998):

\section{"Aunque es parte de la naturaleza, a la cual co-pertenece junto con los demás seres vivos, es distinto de ellos y puede, en virtud de sus especiales características, contraponerse a la misma naturaleza; puede incluso actuar sobre ella, alterar sus procesos y dañarla. Pues es un hecho incontrovertible que el hombre es el único ser vivo capaz de causar deterioros en la naturaleza, algunos de ellos de tal gravedad que amenazan con su misma destrucción".}

La educación forma parte de los elementos simbólicos de la cultura; por ende, es parte de la problemática ambiental, ya que ésta va formando al hombre para que desarrolle una cultura adecuada y acorde a su entorno. Es decir, forma al hombre para encontrar una relación cuidadosa con su medio. Visto así, la educación es el principio de todo conocimiento de tipo conceptual, actitudinal y comportamental, los cuales son la representación del ser y del deber ser dentro de la sociedad en la cual se ha de manifestar la formación de un ciudadano íntegro capaz de convivir en armonía y equilibrio con los demás seres del medio natural que conforman su entorno. En este sentido, entendemos la educación no sólo desde una visión formal, sino también noformal e informal. 
"Para cumplir con esta aspiración, es necesario que los hombres y mujeres posean una cultura general que les permita tomar conciencia y decisiones a favor del medio ambiente" (Andreu y Hernández, 1999). Responsabilidad concierniente a la educación; nuestra formación no está teniendo bases sólidas para un sano vivir, más bien; nuestros estudiantes se preocupan por sacar buenas notas sin entender el contenido, muchas veces, sin saber para qué les va a servir en su vida, no logramos encontrar el punto entre teoría y práctica (esta práctica como vivencia) y lo peor de todo es que se ve reflejado en sus acciones culturales, siendo una cadena que nos ata de pensamiento y acción, imposibilitando una transformación actitudinal,

"se trata de propiciar la construcción de una conciencia ética, para lo cual se debe suscitar en el alumno una reflexión intencionada sobre cómo su aprendizaje se está llevando a cabo, los caminos y procedimientos que ha recorrido, sus aciertos y desaciertos, como también sobre la calidad y validez de los conceptos elaborados, las normas, los valores, métodos, técnicas y actuaciones, sus consecuencias y los impactos generales por las relaciones hombre-sociedad-naturaleza-ciencia-tecnología". (Lineamientos curriculares. Ministerio de Educación Nacional, 1998.).

La propuesta de investigación posee una perspectiva demasiado amplia, puesto que el campo de acción se encuentra dentro del campo educativo: como centro de formación a nivel académico y cultural y, dentro de la sociedad, como ente formador y modelador del comportamiento del hombre, supeditado por la ética y los valores, siendo en estos dos lugares en los que el sujeto permanece durante toda su vida.

En concordancia con lo expuesto anteriormente, se tiene la posibilidad de poder moldear, tomar conciencia y transformar la conducta y la cultura del hombre, para que a partir de sus conocimientos o de su saber actúe tomando parte en la toma de decisiones efectivas en cuanto a los problemas ambientales que hoy día nos afectan considerablemente.

El maestro no puede seguir siendo el especialista que domina, almacena y transmite la información por lo contrario debe ser un dinamizador de procesos que crea espacios, inquietudes y posibilidades para el aprendizaje conjunto de estudiantes, padres de familia y docentes; reflexionan e investigando de manera permanente sobre su quehacer pedagógico y su realidad múltiple y cambiante. (PRAE. Op. Cit. 2008)

Por otro lado, la educación de la ciencia para la acción sociopolítica es ineludiblemente un ejercicio en la clarificación y cambio de valores medioambientales; los problemas no se marcharán por sí solos, ni se resolverán por un rápido apuro técnico mientras se mantenga un estilo de vida libertino, sin que importe el mañana. Se debe de cambiar la manera de pensar, de actuar para que, a partir de este momento, se trasforme la forma de vivir y de comportarnos frente al mundo natural y social, porque el planeta ya no puede sostener nuestro presente estilo de vida. 


\section{BIBLIOGRAFIA.}

Alea, García Alina. (2008) breve historia de la educación ambiental: del conservacionismo al desarrollo sostenible. Ozono mío. Revista de derecho ambiental. $\mathrm{N}^{\circ}$ 7. Extraído el 15 de Agosto de 2008. http://vlex.com.pe/vid/breve-historiaconservacionismo-hacia-41942338

Andreu Gómez Nancy; Hernández Sánchez José Emilio. Importancia de la interdisciplinariedad para una cultura geográfica a favor de la educación. Educación (La Habana) № 98. 1999. Pág. 9 - 16.

Centro Educativo Minuto de Dios Proyecto Ambiental Escolar -PRAE-. “Una estrategia pedagógico - ambiental para la convivencia ciudadana". Ciencias Naturales y Educación Ambiental. Montería. 2008

Corraliza, José Antonio. El comportamiento humano y los problemas ambientales. Estudios de psicología-Psicología Ambiental. V22 № 1. 2001. Pág. 3-10.

González L. de G. Francisco. Producción de conocimiento e identificación de la problemática ambiental, en relación al horizonte dado por el uso de los conceptos de modernidad y posmodernidad. IDEADE. Ambiente y Desarrollo - Ensayos -. 1999. Santa fe de Bogotá, D.C. IDEADE.

Mahecha Clavijo, Germán Roberto. La educación ambiental: ¿solución a un problema o un problema por resolver?. Ambiente y Desarrollo. № 12 Junio. 2003.

Ministerio de Educación Nacional. Lineamientos curriculares. Ciencias Naturales y Educación Ambiental.1998.

Ramírez Restrepo, Rubiel. Ética Ambiental parámetros para una discusión. Universidad de Quindío. Armenia - Quindío1998.

Ruvalcaba Ledesma, Jesús Carlos. Análisis de la práctica docente en la enseñanza de la Ecología. Revista de la universidad del Valle de Atemajac. V16 № 43 Mayo-Agosto. 2002. Pág. 83-87. 